\title{
Palladium-Catalyzed Room Temperature Acylative Cross-Coupling of Activated Amides with Trialkylboranes
}

\author{
Weijia Shi and Gang Zou * \\ School of Chemistry \& Molecular Engineering, East China University of Science \& Technology, 130 Meilong Rd, \\ Shanghai 200237, China; 10111048@mail.ecust.edu.cn \\ * Correspondence: zougang@ecust.edu.cn; Tel.: +86-021-64253881
}

Received: 23 August 2018; Accepted: 18 September 2018; Published: 20 September 2018

\begin{abstract}
A highly efficient acylative cross-coupling of trialkylboranes with activated amides has been effected at room temperature to give the corresponding alkyl ketones in good to excellent yields by using 1,3-bis(2,6-diisopropyl)phenylimidazolylidene and 3-chloropyridine co-supported palladium chloride, the PEPPSI catalyst, in the presence of $\mathrm{K}_{2} \mathrm{CO}_{3}$ in methyl tert-butyl ether. The scope and limitations of the protocol were investigated, showing good tolerance of acyl, cyano, and ester functional groups in the amide counterpart while halo group competed via the classical Suzuki coupling. The trialkylboranes generated in situ by hydroboration of olefins with $\mathrm{BH}_{3}$ or 9-BBN performed similarly to those separately prepared, making this protocol more practical.
\end{abstract}

Keywords: acylative cross-coupling; trialkylborane; amide activation; palladium; $N$-heterocyclic carbene

\section{Introduction}

Amides are unique and ubiquitous substructures in natural and artificial organic functional molecules, because the strong resonance between the carbonyl and amino groups leads to a highly inert and significantly planar linkage. However, the synthetic utility of amides as an acyl source had remained underdeveloped until the seminal publications in 2015 independently from Garg [1], Szostak [2], and ourselves [3], taking advantage of palladium or nickel catalysis for cleavage of electronically or sterically activated amide $\mathrm{C}-\mathrm{N}$ bond, and formation of new carbon-carbon or carbon-oxygen bonds. In the past three years, many efforts have been made to expand the scope of amide counterparts, developing a variety of activated amides suitable for the acylative cross-coupling, e.g., $N$-acyl imides [4-6], $N$-Boc and $N$-Ts /Ms amides [7-15], $N$-acylsaccharins [16-20], and amides of heteroaromatic compounds [21,22]. Comparably, the carbon-centered nucleophile counterparts are still rather undeveloped, in particular, with respect to alkyl ones, although alkyl ketones have been widely found in biologically important molecules and synthetic building blocks for fine chemicals. In fact, besides the closely related esters [23-25], there are only two reports on the acylative cross-coupling of amides with alkyl reagents effected by using nickel catalysts. Garg and co-workers reported the first alkylation of amide derivatives by nickel-catalyzed acylative coupling with alkyl zinc reagents in 2016 [26]. Early this year, Rueping and co-workers described the other nickel-catalyzed cross-coupling of B-alkyl-9-BBN with amides [27]. After extension of the nucleophile counterparts from arylboronic acids to cost-effective diarylborinic acids and tetraarylboronates in palladium-catalyzed acylative cross-coupling of amides [28,29], we are interested in the reactivity of trialkylboranes, which could be readily prepared from alkyl Grignard reagents or olefins via hydroboration. Herein we report a highly efficient palladium-catalyzed acylative cross-coupling of activated amides with trialkylboranes at 
room temperature by using 1,3-bis(2,6-diisopropyl)phenylimidazolylidene (IPr) and 3-chloropyridine (3-ClPy) co-supported palladium chloride, the PEPPSI catalyst developed by Organ et al. [30].

\section{Results and Discussion}

Reaction of 4-methoxy- $N$-methyl- $N$-tosylbenzamide (1a) with the commercially available triethylborane (2a) $\left(\mathrm{BEt}_{3}, 1 \mathrm{M}\right.$ in THF) was chosen as the model to establish an optimal catalyst system for the acyl alkylation of activated amides (Scheme 1).

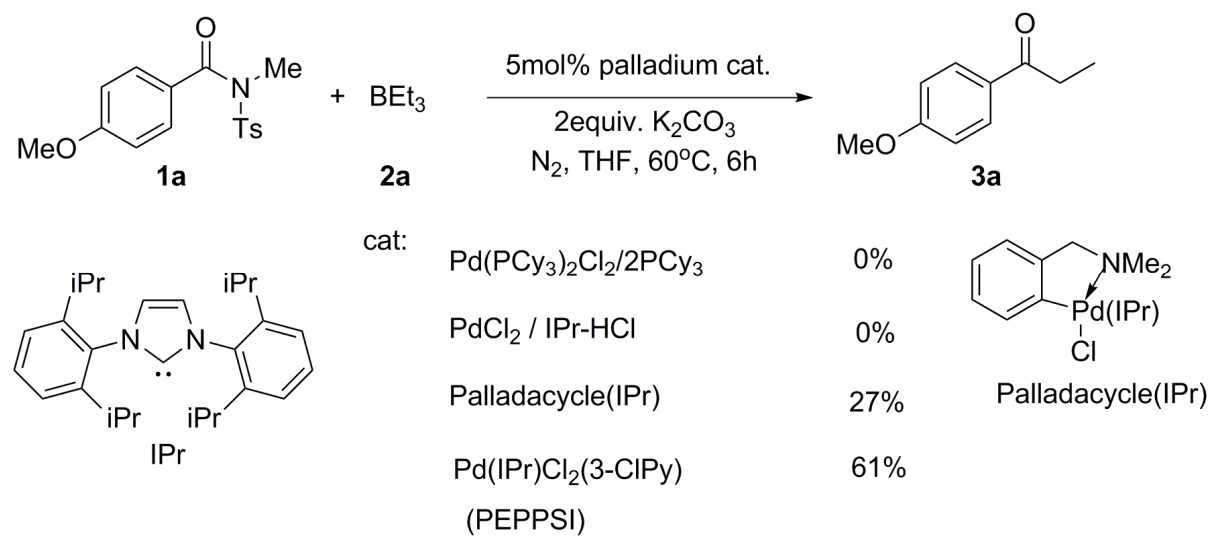

Scheme 1. Screening of palladium catalyst for acyl alkylation of amides with alkylboranes.

The catalyst system $\mathrm{Pd}\left(\mathrm{PCy}_{3}\right)_{2} \mathrm{Cl}_{2} / \mathrm{PCy}_{3}$, which we previously established for acylative cross-coupling of amides with aryl boron compounds, was investigated, at first, in the presence of $\mathrm{K}_{2} \mathrm{CO}_{3}$ as base in THF. Unfortunately, palladium black developed immediately upon heating and no reaction was detected after $6 \mathrm{~h}$ at $60{ }^{\circ} \mathrm{C}$, indicating the incompatibility of the $\mathrm{Pd}\left(\mathrm{PCy}_{3}\right)_{2} \mathrm{Cl}_{2} / \mathrm{PCy}_{3}$ system with the high reducing ability of trialkylboranes. We then turned to the sterically demanding $\mathrm{N}$-heterocyclic carbene (NHC) supported palladium catalysts pioneered by Nolan group [31], for example, 1,3-bis(2,6-diisopropylphenyl)imidazolium chloride (IPr-HCl)/palladium chloride (IPr- $\left.\mathrm{HCl} / \mathrm{PdCl}_{2}\right)$ [32], IPr supported 2-((dimethylamino)methyl)phenyl palladium chloride (Palladacycle(IPr)) [33], and IPr and 3-chloropyridine co-supported PEPPSI catalyst (IPr) $\mathrm{PdCl} 2$ (3-ClPy) [30]. Although the $\mathrm{IPr}-\mathrm{HCl} / \mathrm{PdCl}_{2}$ system also rapidly decomposed to palladium black and lost activity, the palladacycle/IPr and PEPPSI catalysts provided the desired cross-coupling product $3 \mathrm{a}$ in $27 \%$ and $61 \%$ yields, respectively, from 1a with 1.0 equiv $2 \mathrm{a}$ in the presence of 2 equiv $\mathrm{K}_{2} \mathrm{CO}_{3}$. To test if dialkylboranes could be used in the acyl alkylation with amides, we also carried out the model reaction by using diethylborinate $\left(\mathrm{Et}_{2} \mathrm{~B}(\mathrm{OMe}), 1 \mathrm{M}\right.$ in $\left.\mathrm{THF}\right)$ under otherwise identical conditions (Scheme 2). Surprisingly, the C-O cross-coupling product, methyl 4-methoxybenzoate, was obtained in $74 \%$ yield, while the C-C coupling product 3 a was isolated in low $(\sim 5 \%)$ yield, implying a much slower transmetalation of alkyl group from boron to palladium than that of alkoxyl group in dialkylborinates.

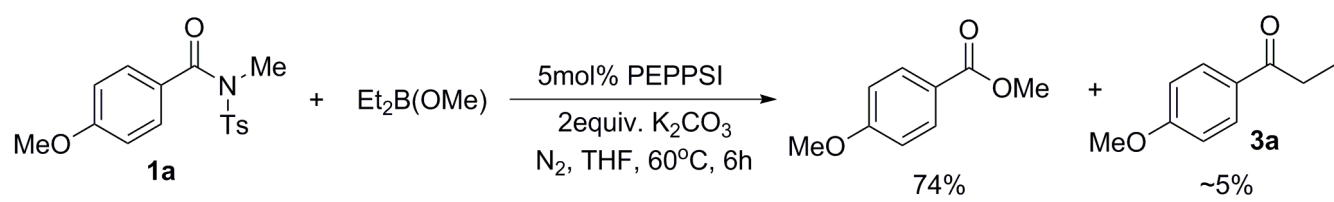

Scheme 2. Unexpected acyl C-O cross-coupling of methyl diethylborinate.

To increase the yield of the desired C-C coupling product in the PEPPSI-catalyzed acyl alkylation of amides, the reaction conditions were optimized with respect to substrate ratio, solvent, base, and reaction temperature, etc. Bases proved to be crucial since no reaction took place in the absence of bases or in the presence of organic base, e.g., $\mathrm{NEt}_{3}$ and pyridine (Table 1, entries 2, 8 and 9). 
The stronger inorganic bases, $\mathrm{Cs}_{2} \mathrm{CO}_{3}(25 \%)$ and $\mathrm{K}_{3} \mathrm{PO}_{4}(33 \%)$, gave the lower yields of $3 a$ than that of $\mathrm{K}_{2} \mathrm{CO}_{3}$, albeit the reaction proceeded faster while the weaker ones, e.g., $\mathrm{Na}_{2} \mathrm{CO}_{3}, \mathrm{NaOAc}$, and $\mathrm{NaHCO}_{3}$, resulted in recovering most of amide substrate $1 \mathbf{a}$ (Table 1, entries 3-7). The yield of 3a could be increased to $80 \%$ with 1.5 equiv $\mathrm{BEt}_{3}$ (Table 1, entry 10). Methyl tert-butyl ether (MTBE) appeared to be the choice of solvent, giving $3 \mathbf{a}$ in $90 \%$ yield (Table 1, entry 12). The reaction occurred even at room temperature and an excellent yield (98\%) for 3a was obtained in $24 \mathrm{~h}$ (Table 1, entry 14). Given the advantages of room temperature organic synthesis [34], we reinvestigated the model reaction in THF or MTBE with $\mathrm{K}_{2} \mathrm{CO}_{3}$ or $\mathrm{K}_{3} \mathrm{PO}_{4}$ as the base (Table 1, entries 15-17). The results confirmed the best performance of the combination of MTBE with $\mathrm{K}_{2} \mathrm{CO}_{3}$.

Table 1. PEPPSI-catalyzed acyl alkylation reaction of amide 1a with triethylborane. ${ }^{\text {a }}$

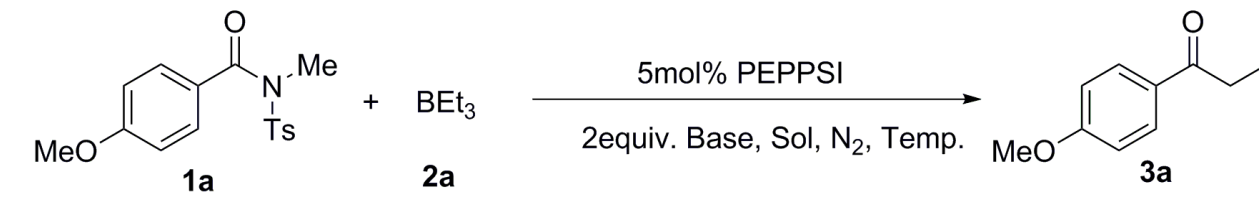

\begin{tabular}{|c|c|c|c|c|c|c|}
\hline Entry & Equiv of $2 a$ & Solvent & Base $^{b}$ & $\mathrm{~T}\left({ }^{\circ} \mathrm{C}\right)$ & Time (h) & Yield $(\%)^{c}$ \\
\hline 1 & 1.1 & $\mathrm{THF}$ & $\mathrm{K}_{2} \mathrm{CO}_{3}$ & 60 & 5 & 61 \\
\hline 2 & 1.1 & THF & / & 60 & 5 & $\mathrm{NR}^{\mathrm{d}}$ \\
\hline 3 & 1.1 & THF & $\mathrm{Cs}_{2} \mathrm{CO}_{3}$ & 60 & 5 & 25 \\
\hline 4 & 1.1 & THF & $\mathrm{K}_{3} \mathrm{PO}_{4}$ & 60 & 5 & 33 \\
\hline 5 & 1.1 & THF & $\mathrm{Na}_{2} \mathrm{CO}_{3}$ & 60 & 8 & 15 \\
\hline 6 & 1.1 & THF & $\mathrm{NaHCO}_{3}$ & 60 & 8 & Trace $^{d}$ \\
\hline 7 & 1.1 & THF & $\mathrm{NaOAc}$ & 60 & 8 & Trace $^{\mathrm{d}}$ \\
\hline 8 & 1.1 & THF & $\mathrm{Et}_{3} \mathrm{~N}$ & 60 & 8 & $\mathrm{NR}^{\mathrm{d}}$ \\
\hline 9 & 1.1 & THF & Pyridine & 60 & 8 & $\mathrm{NR}^{\mathrm{d}}$ \\
\hline 10 & 1.5 & THF & $\mathrm{K}_{2} \mathrm{CO}_{3}$ & 60 & 5 & 80 \\
\hline 11 & 1.5 & Dioxane & $\mathrm{K}_{2} \mathrm{CO}_{3}$ & 60 & 8 & 35 \\
\hline 12 & 1.5 & MTBE & $\mathrm{K}_{2} \mathrm{CO}_{3}$ & 55 & 8 & 90 \\
\hline 13 & 1.5 & $\mathrm{MeCN}$ & $\mathrm{K}_{2} \mathrm{CO}_{3}$ & 60 & 8 & 17 \\
\hline 14 & 1.5 & MTBE & $\mathrm{K}_{2} \mathrm{CO}_{3}$ & $\mathrm{rt}$ & 24 & 98 \\
\hline 15 & 1.5 & MTBE & $\mathrm{K}_{3} \mathrm{PO}_{4}$ & $\mathrm{rt}$ & 24 & 84 \\
\hline 16 & 1.5 & THF & $\mathrm{K}_{2} \mathrm{CO}_{3}$ & $\mathrm{rt}$ & 24 & 82 \\
\hline 17 & 1.5 & $\mathrm{THF}$ & $\mathrm{K}_{3} \mathrm{PO}_{4}$ & $\mathrm{rt}$ & 12 & 40 \\
\hline
\end{tabular}

${ }^{\mathrm{a}}$ Reaction was run at $0.5 \mathrm{~mol}$ scale with respect to $1 \mathrm{1a}^{\mathrm{j}}{ }^{\mathrm{b}} 2$ equiv base used but no base used in Entry 2; ${ }^{\mathrm{c}}$ Isolated yield; ${ }^{\mathrm{d}}$ Amide 1a recovered.

Scope and limitations of the PEPPSI-catalyzed room temperature acylative cross-coupling of activated amides with trialkylboranes were briefly explored (Table 2). Influence of the amide structure was investigated at first under the optimized reaction conditions. Similar to tosyl-activated amide 1a, mesyl (Ms, 1b) analog also reacted efficiently to give 3a in 98\% yield, while tert-butyloxycarbonyl (Boc) activated amide (1c) showed comparably lower reactivity (75\%) (Table 2, entries 1 and 2). Reaction of benzamides bearing an electron-withdrawing group, acyl (1e), cyano (1f), or ester (1g), at the para-position of the benzene ring gave the corresponding ketones $(3 \mathbf{b}, 3 \mathbf{c}$, or $\mathbf{3 d}$ ) in $92 \%, 70 \%$, or 91\% yields, respectively, indicating good functional group compatibility of the PEPPSI-catalyzed acyl alkylation (Table 2, entries 4-6). When 4-chloro- $N$-methyl- $N$-tosylbenzamide (1h) was used as the substrate, the desired acylative cross-coupling product 1-(4-chlorophenyl)propanone was not obtained. Instead, double alkylation product 1-(4-ethylphenyl)propanone (3e) was isolated in 47\% yield, which could be increased to $88 \%$ yield with 2.5 equiv $\mathrm{BEt}_{3}$ (Table 2, entry 7 ), similar to our previous investigation on the palladium-catalyzed acylative Suzuki coupling of arylboronic acids [3]. A small ortho-substituent at the benzene ring appeared to slightly hamper the coupling. In fact, 1-(o-tolyl)propan-1-one (3f) was isolated in 72\% yield from $N$-methyl- $N$-tosyl-2-methylbenzamide (1i) (Table 2, entry 8). Alkyl amides (1j and $\mathbf{1 k}$ ) reacted similarly to give dialkyl ketones $\mathbf{3 g}$ and $\mathbf{3 h}$ in $\mathbf{9 5 \%}$ and $98 \%$ yields, respectively (Table 2, entries 9 and 10). 
Table 2. Scope and limitations of the PEPPSI-catalyzed acyl alkylation reaction of amides with trialkylboranes ${ }^{\text {a }}$.

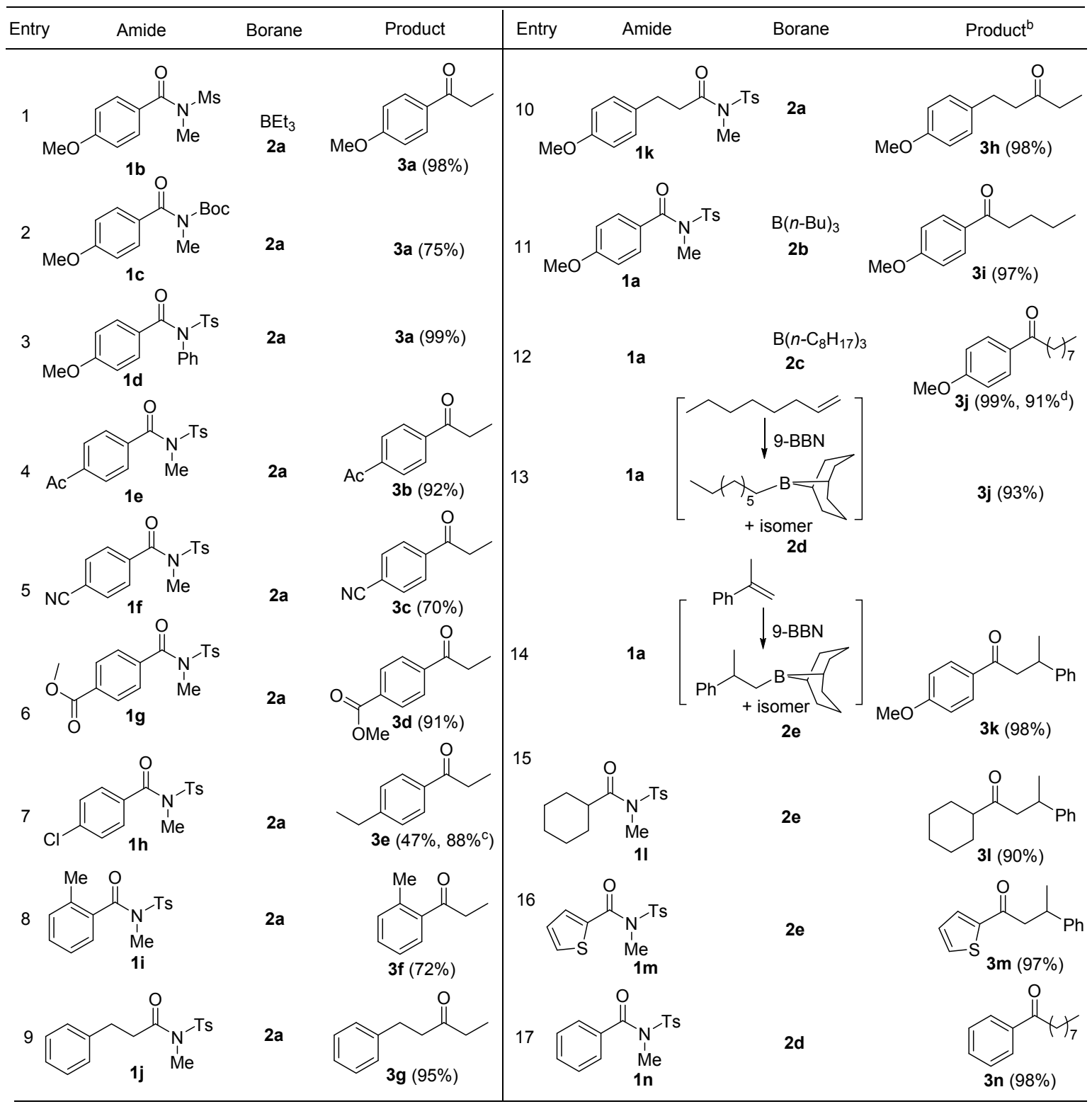

${ }^{a}$ Reaction was run at $0.5 \mathrm{~mol}$ scale with respect to amides with 1.5 equiv trialkylboranes; ${ }^{\mathrm{b}}$ Isolated yield; ${ }^{\mathrm{c}}$ With 2.5 equiv $\mathrm{BEt}_{3} ;{ }^{\mathrm{d}} \mathrm{B}\left(n-\mathrm{C}_{8} \mathrm{H}_{17}\right)_{3}$ and branched isomers in situ generated from hydroboration of octene with 1.0 equiv $\mathrm{BH}_{3}$ in THF at room temperature was used.

Tri(n-butyl)borane $(\mathbf{2} \mathbf{b})$ reacted with 1a similarly to triethylborane (2a) while no reaction of tricyclohexylborane was observed, implying the failure of transmetalation of secondary-alkyl group from boron to palladium. Hydroboration of alkenes represents an alternative route to primary alkyl boranes. However, the long-chain primary alkyl boranes prepared by hydroboration of alkenes are generally contaminated by the presence of branched-isomers due to the non-regiospecific addition of B-H to C-C double bonds [35]. Due to the inertness of the secondary-alkyl group in alkylboranes, reaction of 1a with tri(octyl)boranes prepared by hydroboration of 1-octene proceeded comparably to that of tri( $n$-octyl)borane (2c) from Grignard reagent [36], giving $\mathbf{3 j}$ in $91 \%$ and $99 \%$ yields, respectively (Table 2, entry 12). Given the fact that only one primary alkyl group of trialkylboranes is useful in the palladium-catalyzed acylative cross-coupling, it is more practical to use long-chain alkyl boranes prepared by hydroboration of corresponding olefins by dialkylborane, a more stable B-H source, 
e.g., 9-borabicyclo[3.3.1]nonane (9-BBN). Therefore, we further investigated use of B-octyl-9-BBN formed in situ from octene as the trialkylborane counterpart and obtained $3 \mathbf{j}$ in $93 \%$ yield (Table 2, entry 13). $\beta$-Phenyl propiophenones, which show intrinsic deactivation of the lowest and thermally populated $n, \pi^{*}$-triplet excited states in aryl alkyl ketones [37], could be readily obtained in $98 \%$ yield (3k) by the acyl alkylation of amide 1a with B-(2-phenylpropyl)-9-BBN generated in situ from 9-BBN and $\alpha$-methylstyrene. Alkyl and heterocyclic analogs, e.g., 1-cyclohexyl-3-phenylbutanone (31, 90\%) and 3-phenyl-1-(thiophen-2-yl)butanone (3m, 97\%) could also be prepared efficiently (Table 2, entries 14-16).

However, when amide 1a was subjected to the hydroboration solution of $n$-butyl vinyl ether with 9-BBN the C-O coupling product (vide supra) butyl 4-methoxybenzoate, instead of the desired $\beta$-butoxy-4-methoxypropiophenone, was obtained in $83 \%$ yield, probably due to the facile $\beta$-alkoxy elimination of labile B-(2-butoxyethyl)-9-BBN via intramolecular O-B coordination to B-butoxy-9-BBN for the subsequent $\mathrm{C}-\mathrm{O}$ cross-coupling (Scheme 3).

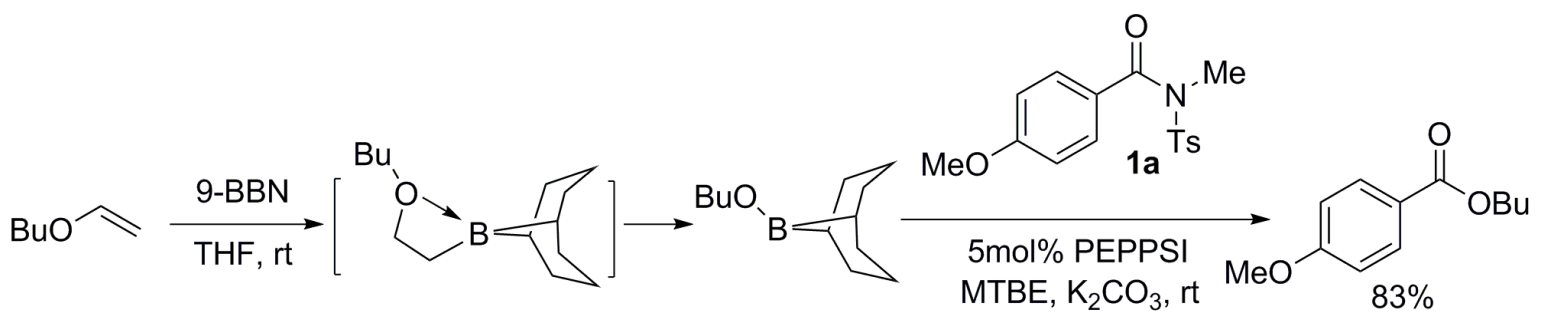

Scheme 3. Formation of borinate and subsequent acyl C-O cross-coupling.

\section{Materials and Methods}

\subsection{General}

Chemicals obtained commercially were used as received. Nuclear magnetic resonance (NMR) spectra were recorded on a Bruker DPX-400 spectrometer (Bruker Co., Billerica, MA, USA) using residue of the deuterated solvent or tetramethylsilane (TMS) as the internal standard (Cambridge Isotope Lab. Inc., Tewksbury, MA, USA). For copies of ${ }^{1} \mathrm{H}$ and ${ }^{13} \mathrm{C}-\mathrm{NMR}$ spectra of the products, please see Supplementary Materials. All products were isolated by flash chromatography using petroleum ether (Sinopharm Chemical Reagent Co. Ltd., Shanghai, China) $\left(60-90{ }^{\circ} \mathrm{C}\right) /$ ethyl acetate (Sinopharm Chemical Reagent Co. Ltd., Shanghai, China) as eluents. Triethylborane (1 M in THF), tributylborane, 9-BBN (1 M in THF) and $\mathrm{BH}_{3}$ (1 M in THF) were purchased from J\&K chemicals (Beijing, China). Amides [3], PEPPSI catalyst [30], 1,3-bis(2,6-diisopropylphenyl)imidazolium chloride (IPr-HCl) [32], palladacycle (IPr) [33], and tri(n-octyl)borane [36] were prepared according to the procedures reported previously. Solvents, methyl tert-butyl ether (MTBE), tetrahydrofuran (THF), and dioxane were dried over sodium while acetonitrile was distilled over $\mathrm{CaH}_{2}$ prior to use.

\subsection{General Procedure for the PEPPSI-Catalyzed Cross-Coupling of N-Methyl-N-Tosylamides with Trialkylboranes}

A Schlenk tube $(20 \mathrm{~mL})$ charged with amide $(0.5 \mathrm{mmol})$, PEPPSI $(0.025 \mathrm{mmol}, 5 \mathrm{~mol} \%)$, and $\mathrm{K}_{2} \mathrm{CO}_{3}$ ( 2 equiv) was degassed and then refilled with nitrogen, three times. Then, solvent MTBE $(6.0 \mathrm{~mL})$ was added via syringe followed by $0.75 \mathrm{~mL}$ (1.5 equiv) borane solution ( $1 \mathrm{M}$ in THF), which was commercially available or was in situ prepared by hydroboration of olefin. The resulted mixture was stirred for $24 \mathrm{~h}$ at room temperature under $\mathrm{N}_{2}$ atmosphere. The reaction was quenched with iced water $(5 \mathrm{~mL})$ and extracted with MTBE $(2 \times 5 \mathrm{~mL})$. The combined MTBE extracts were dried over anhydrous $\mathrm{Na}_{2} \mathrm{SO}_{4}$. After filtration, solvents were removed by rotavapor to afford the crude product, which was purified by flash column chromatography on silica gel using petroleum ether/EtOAc as the eluents.

1-(4-Methoxyphenyl)propanone (3a) [38]: Colorless oil (80.3 mg, 98\%). ${ }^{1} \mathrm{H}-\mathrm{NMR}\left(400 \mathrm{MHz}, \mathrm{CDCl}_{3}\right)$ $\delta(\mathrm{ppm}): 7.91$ (d, J = 8.8 Hz, 2H, Ar), 6.89 (d, J = 8.8 Hz, 2H, Ar), $3.82(\mathrm{~s}, 3 \mathrm{H}, \mathrm{OMe}), 2.91(\mathrm{q}, J=7.2 \mathrm{~Hz}$, 
$\left.2 \mathrm{H}, \mathrm{CH}_{2}\right), 1.18\left(\mathrm{t}, J=7.2 \mathrm{~Hz}, 3 \mathrm{H}, \mathrm{CH}_{3}\right) .{ }^{13} \mathrm{C}-\mathrm{NMR}\left(100 \mathrm{MHz}, \mathrm{CDCl}_{3}\right) \delta(\mathrm{ppm}): 199.34(\mathrm{C}=\mathrm{O}), 163.16$ $\left(\mathrm{C}^{4} \mathrm{Ar}\right), 130.06\left(\mathrm{C}^{2,6} \mathrm{Ar}\right), 129.85\left(\mathrm{C}^{1} \mathrm{Ar}\right), 113.52\left(\mathrm{C}^{3,5} \mathrm{Ar}\right), 55.29(\mathrm{OMe}), 31.26\left(\mathrm{CH}_{2}\right), 8.29\left(\mathrm{CH}_{3}\right)$.

1-(4-Acetylphenyl)propanone (3b) [39]: White powder (80.9 mg, 92\%), mp 69-71 ${ }^{\circ} \mathrm{C} .{ }^{1} \mathrm{H}-\mathrm{NMR}(400 \mathrm{MHz}$, $\left.\mathrm{CDCl}_{3}\right) \delta(\mathrm{ppm}): 8.00(\mathrm{~s}, 4 \mathrm{H}, \mathrm{Ar}), 3.01\left(\mathrm{q}, J=7.2 \mathrm{~Hz}, 2 \mathrm{H}, \mathrm{CH}_{2}\right), 2.62(\mathrm{~s}, 3 \mathrm{H}, \mathrm{Ac}), 1.21(\mathrm{t}, J=7.2 \mathrm{~Hz}$, $\left.3 \mathrm{H}, \mathrm{CH}_{3}\right) \cdot{ }^{13} \mathrm{C}-\mathrm{NMR}\left(100 \mathrm{MHz}, \mathrm{CDCl}_{3}\right) \delta(\mathrm{ppm}): 200.13(\mathrm{C}=\mathrm{O}), 197.46(\mathrm{C}=\mathrm{O}), 139.96\left(\mathrm{C}^{1 / 4} \mathrm{Ar}\right), 139.90$ $\left(\mathrm{C}^{1 / 4} \mathrm{Ar}\right), 128.41\left(\mathrm{C}_{\mathrm{Ar}}\right), 128.07\left(\mathrm{C}_{\mathrm{Ar}}\right), 32.15\left(\mathrm{CH}_{2}\right), 26.81(\mathrm{MeCO}), 7.97\left(\mathrm{CH}_{3}\right)$.

4-Propionylbenzonitrile (3c) [40]: White powder (55.8 mg, 70\%), mp 53-55 ${ }^{\circ} \mathrm{C} .{ }^{1} \mathrm{H}-\mathrm{NMR}(400 \mathrm{MHz}$, $\left.\mathrm{CDCl}_{3}\right) \delta(\mathrm{ppm}): 7.97(\mathrm{~d}, J=8.4 \mathrm{~Hz}, 2 \mathrm{H}, \mathrm{Ar}), 7.69(\mathrm{~d}, J=8.4 \mathrm{~Hz}, 2 \mathrm{H}, \mathrm{Ar}), 2.95\left(\mathrm{q}, J=7.2 \mathrm{~Hz}, 2 \mathrm{H}, \mathrm{CH}_{2}\right)$, $1.15\left(\mathrm{t}, J=7.2 \mathrm{~Hz}, 3 \mathrm{H}, \mathrm{CH}_{3}\right) .{ }^{13} \mathrm{C}-\mathrm{NMR}\left(100 \mathrm{MHz}, \mathrm{CDCl}_{3}\right) \delta(\mathrm{ppm}): 199.16(\mathrm{C}=\mathrm{O}), 139.64\left(\mathrm{C}^{4} \mathrm{Ar}\right), 132.33$ $\left(\mathrm{C}_{\mathrm{Ar}}\right), 128.23\left(\mathrm{C}_{\mathrm{Ar}}\right), 117.84\left(\mathrm{CN} / \mathrm{C}^{1} \mathrm{Ar}\right), 115.95\left(\mathrm{CN} / \mathrm{C}^{1} \mathrm{Ar}\right), 32.01\left(\mathrm{CH}_{2}\right), 7.75\left(\mathrm{CH}_{3}\right)$.

Methyl 4-propionylbenzoate (3d) [41]: White crystalline powder, (87.1 mg, 91\%), mp 81-83 ${ }^{\circ} \mathrm{C} .{ }^{1} \mathrm{H}-\mathrm{NMR}$ $\left(400 \mathrm{MHz}, \mathrm{CDCl}_{3}\right) \delta(\mathrm{ppm}): 8.08(\mathrm{~d}, J=8.4 \mathrm{~Hz}, 2 \mathrm{H}, \mathrm{Ar}), 7.97(\mathrm{~d}, J=8.4 \mathrm{~Hz}, 2 \mathrm{H}, \mathrm{Ar}), 3.92(\mathrm{~s}, 3 \mathrm{H}, \mathrm{OMe})$, $3.05\left(\mathrm{q}, J=7.2 \mathrm{~Hz}, 2 \mathrm{H}, \mathrm{CH}_{2}\right), 1.21\left(\mathrm{t}, J=7.2 \mathrm{~Hz}, 3 \mathrm{H}, \mathrm{CH}_{3}\right) .{ }^{13} \mathrm{C}-\mathrm{NMR}\left(100 \mathrm{MHz}, \mathrm{CDCl}_{3}\right) \delta(\mathrm{ppm}): 200.15$ $(\mathrm{C}=\mathrm{O}), 166.17(\mathrm{OC}=\mathrm{O}), 140.01\left(\mathrm{C}^{1 / 4}{ }_{\mathrm{Ar}}\right), 133.59\left(\mathrm{C}^{1 / 4} \mathrm{Ar}\right), 129.72\left(\mathrm{C}_{\mathrm{Ar}}\right), 127.78\left(\mathrm{C}_{\mathrm{Ar}}\right), 52.35(\mathrm{OMe}), 32.10$ $\left(\mathrm{CH}_{2}\right), 7.96\left(\mathrm{CH}_{3}\right)$.

1-(4-Ethylphenyl)propanone (3e) [42]: Colorless oil (71.1 mg, 88\%, with 2.5 equiv BEt $\left.{ }_{3}\right) .{ }^{1} \mathrm{H}-\mathrm{NMR}(400$ $\left.\mathrm{MHz}, \mathrm{CDCl}_{3}\right) \delta(\mathrm{ppm}): 7.81(\mathrm{~d}, J=8.4 \mathrm{~Hz}, 2 \mathrm{H}, \mathrm{Ar}), 7.19(\mathrm{~d}, J=8.4 \mathrm{~Hz}, 2 \mathrm{H}, \mathrm{Ar}), 2.90(\mathrm{q}, J=7.2 \mathrm{~Hz}, 2 \mathrm{H}$, $\left.\mathrm{COCH}_{2}\right), 2.61\left(\mathrm{q}, J=7.6 \mathrm{~Hz}, 2 \mathrm{H}, \mathrm{ArCH}_{2}\right), 1.17\left(\mathrm{t}, J=7.6 \mathrm{~Hz}, 3 \mathrm{H}, \mathrm{COCH}_{2} \mathrm{CH}_{3}\right), 1.13(\mathrm{t}, J=7.2 \mathrm{~Hz}, 3 \mathrm{H}$, $\left.\mathrm{Ar} \mathrm{CH}_{2} \mathrm{CH}_{3}\right) .{ }^{13} \mathrm{C}-\mathrm{NMR}\left(100 \mathrm{MHz}, \mathrm{CDCl}_{3}\right) \delta(\mathrm{ppm}): 200.51(\mathrm{C}=\mathrm{O}), 149.71\left(\mathrm{C}^{4} \mathrm{Ar}\right), 134.59\left(\mathrm{C}^{1} \mathrm{Ar}\right), 128.14$ $\left(\mathrm{C}_{\mathrm{Ar}}\right), 127.97\left(\mathrm{C}_{\mathrm{Ar}}\right), 31.60\left(\mathrm{COCH}_{2}\right), 28.85\left(\mathrm{ArCH}_{2}\right), 15.16\left(\mathrm{ArCH}_{2} \mathrm{CH}_{3}\right), 8.27\left(\mathrm{COCH}_{2} \mathrm{CH}_{3}\right)$.

1-(o-Tolyl)propanone (3f) [43]: Colorless oil (53.4 mg, 72\%). ${ }^{1} \mathrm{H}-\mathrm{NMR}\left(400 \mathrm{MHz}, \mathrm{CDCl}_{3}\right) \delta(\mathrm{ppm}): 7.53$ (d, $J=7.6 \mathrm{~Hz}, 1 \mathrm{H}), 7.29-7.25(\mathrm{~m}, 1 \mathrm{H}, \mathrm{Ar}), 7.16-7.14(\mathrm{~m}, 2 \mathrm{H}, \mathrm{Ar}), 2.82\left(\mathrm{q}, J=7.2 \mathrm{~Hz}, 2 \mathrm{H}, \mathrm{CH}_{2}\right), 2.41$ $\left(\mathrm{s}, 3 \mathrm{H}, \mathrm{ArCH}_{3}\right), 1.11\left(\mathrm{t}, J=7.2 \mathrm{~Hz}, 3 \mathrm{H}, \mathrm{CH}_{3}\right) .{ }^{13} \mathrm{C}-\mathrm{NMR}\left(100 \mathrm{MHz}, \mathrm{CDCl}_{3}\right) \delta(\mathrm{ppm}): 205.04(\mathrm{C}=\mathrm{O})$, $138.01\left(\mathrm{C}^{1 / 2} \mathrm{Ar}\right), 137.76\left(\mathrm{C}^{1 / 2} \mathrm{Ar}\right), 131.81\left(\mathrm{C}_{\mathrm{Ar}}\right), 130.97\left(\mathrm{C}_{\mathrm{Ar}}\right), 128.19\left(\mathrm{C}_{\mathrm{Ar}}\right), 125.56\left(\mathrm{C}_{\mathrm{Ar}}\right), 34.64\left(\mathrm{CH}_{2}\right), 21.19$ $\left(\mathrm{ArCH}_{3}\right), 8.31\left(\mathrm{CH}_{3}\right)$.

1-Phenylpentan-3-one (3g) [44]: Colorless oil (77.1 mg, 95\%). ${ }^{1} \mathrm{H}-\mathrm{NMR}\left(400 \mathrm{MHz}, \mathrm{CDCl}_{3}\right) \delta(\mathrm{ppm})$ : 7.33-7.29 (m, 2H, Ph), 7.23-7.20 (m, 3H, Ph), $2.93\left(\mathrm{t}, J=7.6 \mathrm{~Hz}, 2 \mathrm{H}, \mathrm{PhCH}_{2} \mathrm{CH}_{2} \mathrm{CO}\right), 2.76(\mathrm{t}, J=7.6 \mathrm{~Hz}, 2 \mathrm{H}$, $\left.\mathrm{PhCH}_{2}\right), 2.43\left(\mathrm{q}, J=7.2 \mathrm{~Hz}, 2 \mathrm{H}, \mathrm{CO} \mathrm{CH}_{2} \mathrm{CH}_{3}\right), 1.07\left(\mathrm{t}, J=7.2 \mathrm{~Hz}, 3 \mathrm{H}, \mathrm{CH}_{3}\right) \cdot{ }^{13} \mathrm{C}-\mathrm{NMR}\left(100 \mathrm{MHz}, \mathrm{CDCl}_{3}\right)$ $\delta(\mathrm{ppm}): 210.61(\mathrm{C}=\mathrm{O}), 141.10\left(\mathrm{C}^{1} \mathrm{Ph}\right), 128.41(\mathrm{Ph}), 128.24(\mathrm{Ph}), 126.00\left(\mathrm{C}^{4} \mathrm{Ph}\right), 43.81\left(\mathrm{PhCH}_{2} \mathrm{CH}_{2} \mathrm{CO}\right)$, $36.05\left(\mathrm{COCH}_{2} \mathrm{CH}_{3}\right), 29.77\left(\mathrm{PhCH}_{2}\right), 7.69\left(\mathrm{CH}_{3}\right)$.

1-(4-Methoxyphenyl)pentan-3-one (3h) [45]: Colorless oil (94.3 mg, 98\%). ${ }^{1} \mathrm{H}-\mathrm{NMR}\left(400 \mathrm{MHz}, \mathrm{CDCl}_{3}\right)$ $\delta(\mathrm{ppm}): 7.09(\mathrm{~d}, J=8.8 \mathrm{~Hz}, 2 \mathrm{H}, \mathrm{Ar}), 6.81(\mathrm{~d}, J=8.8 \mathrm{~Hz}, 2 \mathrm{H}, \mathrm{Ar}), 3.77(\mathrm{~s}, 3 \mathrm{H}, \mathrm{OMe}), 2.84(\mathrm{t}, J=7.6 \mathrm{~Hz}, 2 \mathrm{H}$, $\left.\mathrm{PhCH}_{2} \mathrm{CH}_{2} \mathrm{CO}\right), 2.64\left(\mathrm{t}, J=7.6 \mathrm{~Hz}, 2 \mathrm{H}, \mathrm{PhCH}_{2}\right), 2.39\left(\mathrm{q}, J=7.6 \mathrm{~Hz}, 2 \mathrm{H}, \mathrm{COCH}_{2} \mathrm{CH}_{3}\right), 1.03(\mathrm{t}, J=7.6 \mathrm{~Hz}$, $\left.3 \mathrm{H}, \mathrm{CH}_{3}\right) .{ }^{13} \mathrm{C}-\mathrm{NMR}\left(100 \mathrm{MHz}, \mathrm{CDCl}_{3}\right) \delta(\mathrm{ppm}): 210.80(\mathrm{C}=\mathrm{O}), 157.85\left(\mathrm{C}^{4} \mathrm{Ar}\right), 133.12\left(\mathrm{C}^{1} \mathrm{Ar}\right), 129.16$ $\left(\mathrm{C}_{\mathrm{Ar}}\right), 113.79\left(\mathrm{C}_{\mathrm{Ar}}\right), 55.17(\mathrm{OMe}), 44.09\left(\mathrm{PhCH}_{2} \mathrm{CH}_{2} \mathrm{CO}\right), 36.08\left(\mathrm{COCH}_{2} \mathrm{CH}_{3}\right), 28.93\left(\mathrm{PhCH}_{2}\right), 7.68\left(\mathrm{CH}_{3}\right)$.

1-(4-Methoxyphenyl)pentanone (3i) [46]: Colorless oil (93.5 mg, 97\%). ${ }^{1} \mathrm{H}-\mathrm{NMR}\left(400 \mathrm{MHz}, \mathrm{CDCl}_{3}\right)$ $\delta(\mathrm{ppm}): 7.93(\mathrm{~d}, J=8.8 \mathrm{~Hz}, 2 \mathrm{H}, \mathrm{Ar}), 6.91(\mathrm{~d}, J=9.2 \mathrm{~Hz}, 2 \mathrm{H}, \mathrm{Ar}), 3.85(\mathrm{~s}, 3 \mathrm{H}, \mathrm{OMe}), 2.90(\mathrm{t}, J=7.6 \mathrm{~Hz}$, $\left.2 \mathrm{H}, \mathrm{COCH}_{2}\right), 1.73-1.66\left(\mathrm{~m}, 2 \mathrm{H}, \mathrm{COCH}_{2} \mathrm{CH}_{2}\right), 1.44-1.34\left(\mathrm{~m}, 2 \mathrm{H}, \mathrm{COCH}_{2} \mathrm{CH}_{2} \mathrm{CH}_{2}\right), 0.94(\mathrm{t}, J=7.6 \mathrm{~Hz}$, 3H). ${ }^{13} \mathrm{C}-\mathrm{NMR}\left(100 \mathrm{MHz}, \mathrm{CDCl}_{3}\right) \delta(\mathrm{ppm}): 199.20(\mathrm{C}=\mathrm{O}), 163.23\left(\mathrm{C}^{4} \mathrm{Ar}\right), 130.25\left(\mathrm{C}^{2,6} \mathrm{Ar}\right), 130.11\left(\mathrm{C}^{1} \mathrm{Ar}\right)$, $113.59\left(\mathrm{C}^{3,5} \mathrm{Ar}\right), 55.37(\mathrm{OMe}), 37.95\left(\mathrm{COCH}_{2}\right), 26.68\left(\mathrm{CH}_{2}\right), 22.48\left(\mathrm{CH}_{2}\right), 13.90\left(\mathrm{CH}_{3}\right)$.

1-(4-Methoxyphenyl)nonanone (3j) [47]: Colorless oil (123.0 mg, 99\%, with tri(n-octyl)borane; $115 \mathrm{mg}$, $93 \%$ with hydroboration of octene with $9-\mathrm{BBN} ; 113 \mathrm{mg}, 91 \%$ with hydroboration of octene with $\mathrm{BH}_{3}$. ${ }^{1} \mathrm{H}-\mathrm{NMR}\left(400 \mathrm{MHz}, \mathrm{CDCl}_{3}\right) \delta(\mathrm{ppm}): 7.86(\mathrm{~d}, J=9.2 \mathrm{~Hz}, 2 \mathrm{H}, \mathrm{Ar}), 6.84(\mathrm{~d}, J=9.2 \mathrm{~Hz}, 2 \mathrm{H}, \mathrm{Ar}), 3.78(\mathrm{~s}, 3 \mathrm{H}$, $\mathrm{OMe}), 2.82\left(\mathrm{t}, J=7.6 \mathrm{~Hz}, 2 \mathrm{H}, \mathrm{COCH}_{2}\right), 1.63\left(\mathrm{t}, J=7.6 \mathrm{~Hz}, 2 \mathrm{H}, \mathrm{COCH}_{2} \mathrm{CH}_{2}\right), 1.29-1.19\left(\mathrm{~m}, 10 \mathrm{H},\left(\mathrm{CH}_{2}\right)_{5}\right)$, $0.80\left(\mathrm{t}, J=7.2 \mathrm{~Hz}, 3 \mathrm{H}, \mathrm{CH}_{3}\right) \cdot{ }^{13} \mathrm{C}-\mathrm{NMR}\left(100 \mathrm{MHz}, \mathrm{CDCl}_{3}\right) \delta(\mathrm{ppm}): 199.20(\mathrm{C}=\mathrm{O}), 163.23\left(\mathrm{C}^{4} \mathrm{Ar}\right), 130.25$ 
$\left(\mathrm{C}^{2,6} \mathrm{Ar}\right), 130.13\left(\mathrm{C}^{1} \mathrm{Ar}\right), 113.60\left(\mathrm{C}^{3,5} \mathrm{Ar}\right), 55.37(\mathrm{OMe}), 38.26\left(\mathrm{COCH}_{2}\right), 31.79\left(\mathrm{CH}_{2}\right), 29.41\left(\mathrm{CH}_{2}\right), 29.39$ $\left(\mathrm{CH}_{2}\right), 29.14\left(\mathrm{CH}_{2}\right), 24.59\left(\mathrm{CH}_{2}\right), 22.61\left(\mathrm{CH}_{2}\right), 14.05\left(\mathrm{CH}_{3}\right)$.

1-(4-Methoxyphenyl)-3-phenylbutanone (3k) [46]: White powder (125.0 mg, 98\%), mp 84-86 ${ }^{\circ} \mathrm{C} .{ }^{1} \mathrm{H}-\mathrm{NMR}$ $\left(400 \mathrm{MHz}, \mathrm{CDCl}_{3}\right) \delta(\mathrm{ppm}): 7.93(\mathrm{~d}, \mathrm{~J}=8.8 \mathrm{~Hz}, 2 \mathrm{H}, \mathrm{Ar}), 7.34-7.30(\mathrm{~m}, 4 \mathrm{H}, \mathrm{Ph}), 7.23-7.19(\mathrm{~m}, 1 \mathrm{H}, \mathrm{Ph})$, $6.92(\mathrm{~d}, J=8.8 \mathrm{~Hz}, 2 \mathrm{H}, \mathrm{Ar}), 3.86(\mathrm{~s}, 3 \mathrm{H}, \mathrm{OMe}), 3.55-3.47(\mathrm{~m}, 1 \mathrm{H}, \mathrm{CH}), 3.26\left(\mathrm{dd}, J_{1}=16.4 \mathrm{~Hz}, J_{2}=5.6 \mathrm{~Hz}\right.$, $\left.1 \mathrm{H}, \mathrm{CH}_{2}\right), 3.14\left(\mathrm{dd}, J_{1}=16.0 \mathrm{~Hz}, J_{2}=8.4 \mathrm{~Hz}, 1 \mathrm{H}, \mathrm{CH}_{2}\right), 1.34\left(\mathrm{~d}, J=7.8,3 \mathrm{H}, \mathrm{CH}_{3}\right) .{ }^{13} \mathrm{C}-\mathrm{NMR}(100 \mathrm{MHz}$, $\left.\mathrm{CDCl}_{3}\right) \delta(\mathrm{ppm}): 197.59(\mathrm{C}=\mathrm{O}), 163.31\left(\mathrm{C}^{4} \mathrm{Ar}\right), 146.65\left(\mathrm{C}_{\mathrm{Ph}}^{1}\right), 130.28\left(\mathrm{C}_{\mathrm{Ar}} / \mathrm{C}_{\mathrm{Ph}}\right), 130.21\left(\mathrm{C}^{1} \mathrm{Ar}\right), 128.43$ $\left(\mathrm{C}_{\mathrm{Ph}}\right), 126.79\left(\mathrm{C}_{\mathrm{Ph}}\right), 126.15\left(\mathrm{C}^{4} \mathrm{Ph}\right), 113.61\left(\mathrm{C}^{3,5} \mathrm{Ar}\right), 55.37(\mathrm{OMe}), 46.59\left(\mathrm{COCH}_{2}\right), 35.67(\mathrm{CH}), 21.79\left(\mathrm{CH}_{3}\right)$.

1-Cyclohexyl-3-phenylbutanone (31): Colorless oil (103.7 mg, 90\%). ${ }^{1} \mathrm{H}-\mathrm{NMR}\left(400 \mathrm{MHz}, \mathrm{CDCl}_{3}\right) \delta(\mathrm{ppm})$ : 7.33-7.29 (m, 2H, Ph), 7.25-7.19 (m, 3H, Ph), 3.41-3.32 (m, 1H, PhCH), $2.77\left(\mathrm{dd}, J_{1}=16.8 \mathrm{~Hz}, J_{2}=6.4 \mathrm{~Hz}\right.$, $1 \mathrm{H}, \mathrm{CO} \mathrm{CH}), 2.69\left(\mathrm{dd}, J_{1}=16.4 \mathrm{~Hz}, J_{2}=8.0 \mathrm{~Hz}, 1 \mathrm{H}, \mathrm{COCH}_{2}\right), 2.29-2.22(\mathrm{~m}, 1 \mathrm{H}, \mathrm{COCH}), 1.83-1.65(\mathrm{~m}$, $5 \mathrm{H}, \mathrm{Cy}), 1.35-1.17\left(\mathrm{~m}, 8 \mathrm{H}, \mathrm{Cy}\right.$ overlapped with $\left.\mathrm{CH}_{3}\right) .{ }^{13} \mathrm{C}-\mathrm{NMR}\left(100 \mathrm{MHz}, \mathrm{CDCl}_{3}\right) \delta(\mathrm{ppm}): 212.83$ $(\mathrm{C}=\mathrm{O}), 146.54\left(\mathrm{C}^{1} \mathrm{Ph}\right), 128.39\left(\mathrm{C}_{\mathrm{Ph}}\right), 126.75\left(\mathrm{C}_{\mathrm{Ph}}\right), 126.11\left(\mathrm{C}^{4} \mathrm{Ph}\right), 51.19(\mathrm{COCH}), 49.10\left(\mathrm{COCH}_{2}\right), 35.02$ $(\mathrm{CH}), 28.22(\mathrm{Cy}), 28.05(\mathrm{Cy}), 25.78(\mathrm{Cy}), 25.61(\mathrm{Cy}), 25.53(\mathrm{Cy}), 21.79\left(\mathrm{CH}_{3}\right)$. HRMS (ESI) $\mathrm{m} / z$ calcd for $\mathrm{C}_{16} \mathrm{H}_{22} \mathrm{ONa}[\mathrm{M}+\mathrm{Na}]^{+} 253.1568$, found 253.1569 .

3-Phenyl-1-(thiophen-2-yl)butanone (3m) [48]: Colorless oil (111.5 mg, 97\%). ${ }^{1} \mathrm{H}-\mathrm{NMR}\left(400 \mathrm{MHz}, \mathrm{CDCl}_{3}\right)$ $\delta(\mathrm{ppm}):$ 7.70-7.69 $(\mathrm{m}, 1 \mathrm{H}$, thiophenyl), 7.64-7.63 (m, 1H, thiophenyl), 7.36-7.28 $(\mathrm{m}, 4 \mathrm{H}, \mathrm{Ph}), 7.25-7.23$ $(\mathrm{m}, 1 \mathrm{H}, \mathrm{Ph}), 7.14-7.12\left(\mathrm{~m}, 1 \mathrm{H}\right.$, thiophenyl), 3.56-3.51 (m, 1H, PhCH), $3.24\left(\mathrm{dd}, J_{1}=16.0 \mathrm{~Hz}, J_{2}=6.0 \mathrm{~Hz}\right.$, $\left.1 \mathrm{H}, \mathrm{CH}_{2} \mathrm{CO}\right), 3.14\left(\mathrm{dd}, J_{1}=15.6 \mathrm{~Hz}, J_{2}=8.4 \mathrm{~Hz}, 1 \mathrm{H}, \mathrm{CH}_{2} \mathrm{CO}\right), 1.38\left(\mathrm{~d}, J=6.8 \mathrm{~Hz}, 3 \mathrm{H}, \mathrm{CH}_{3}\right) .{ }^{13} \mathrm{C}-\mathrm{NMR}$ $\left(100 \mathrm{MHz}, \mathrm{CDCl}_{3}\right) \delta(\mathrm{ppm}): 191.94(\mathrm{C}=\mathrm{O}), 146.18\left(\mathrm{C}^{1} \mathrm{Ph}\right), 144.60$ ( $\mathrm{C}^{2}$ thiophenyl), 133.56 (thiophenyl), 131.81 (thiophenyl), $128.49\left(\mathrm{C}_{\mathrm{Ph}}\right), 128.01$ (thiophenyl), $126.78\left(\mathrm{C}_{\mathrm{Ph}}\right), 126.30\left(\mathrm{C}^{4} \mathrm{Ph}\right), 47.78\left(\mathrm{COCH}_{2}\right), 35.93$ (CH), $21.64\left(\mathrm{CH}_{3}\right)$.

1-Phenylnonanone (3n) [47]: Colorless oil (106.8 mg, 98\%). ${ }^{1} \mathrm{H}-\mathrm{NMR}\left(400 \mathrm{MHz}, \mathrm{CDCl}_{3}\right) \delta(\mathrm{ppm}): 7.97-7.95$ $(\mathrm{m}, 2 \mathrm{H}, \mathrm{Ph}), 7.57-7.53(\mathrm{~m}, 1 \mathrm{H}, \mathrm{Ph}), 7.47-7.44(\mathrm{~m}, 2 \mathrm{H}, \mathrm{Ph}), 2.96\left(\mathrm{t}, J=7.6 \mathrm{~Hz}, 2 \mathrm{H}, \mathrm{COCH}_{2}\right), 1.77-1.69$ $\left(\mathrm{m}, 2 \mathrm{H}, \mathrm{COCH}_{2} \mathrm{CH}_{2}\right), 1.39-1.25\left(\mathrm{~m}, 10 \mathrm{H},\left(\mathrm{CH}_{2}\right)_{5}\right), 0.88\left(\mathrm{t}, J=6.8 \mathrm{~Hz}, 3 \mathrm{H}, \mathrm{CH}_{3}\right) .{ }^{13} \mathrm{C}-\mathrm{NMR}(100 \mathrm{MHz}$, $\left.\left.\mathrm{CDCl}_{3}\right) \delta(\mathrm{ppm}): 200.62(\mathrm{C}=\mathrm{O}), 137.05 \mathrm{C}_{\mathrm{Ph}}^{1}\right), 132.83\left(\mathrm{C}_{\mathrm{Ph}}^{4}\right), 128.51\left(\mathrm{C}_{\mathrm{Ph}}\right), 128.02\left(\mathrm{C}_{\mathrm{Ph}}\right), 38.61\left(\mathrm{COCH}_{2}\right)$, $31.81\left(\mathrm{CH}_{2}\right), 29.42\left(\mathrm{CH}_{2}\right), 29.36\left(\mathrm{CH}_{2}\right), 29.15\left(\mathrm{CH}_{2}\right), 24.36\left(\mathrm{CH}_{2}\right), 22.63\left(\mathrm{CH}_{2}\right), 14.08\left(\mathrm{CH}_{3}\right)$.

\section{Conclusions}

In summary, we have developed a highly efficient acylative cross-coupling of trialkylboranes with activated amides by using 1,3-bis(2,6-diisopropyl)phenylimidazolylidene and 3-chloropyridine co-supported palladium chloride, the PEPPSI catalyst, under mild conditions. Bases appeared to play a key role in the reaction, among which $\mathrm{K}_{2} \mathrm{CO}_{3}$ performed best in MTBE at room temperature. The reaction proceeded to give alkyl ketones in good to excellent yields, tolerating a variety of functional groups in the amide counterpart. Unlike the high-order arylboron compounds, in which all the aryl groups react effectively, only one of the three primary alkyl groups in trialkylboranes could be used as alkyl source for the acyl alkylation. The trialkylboranes generated in situ by hydroboration of olefins with $\mathrm{BH}_{3}$ or 9-BBN performed comparably to those separately prepared. This protocol complements ketone synthesis via palladium-catalyzed acylative cross-coupling of amides, providing a feasible access to both monoalkyl and dialkyl ketones.

Supplementary Materials: The following are available online, Figures S1-S28: ${ }^{1} \mathrm{H}$ and ${ }^{13} \mathrm{C}-\mathrm{NMR}$ of products 3a-3n, Figure S29 HRMS of 31.

Author Contributions: Conceptualization, G.Z.; Investigation, W.S.; Writing-Original Draft Preparation, W.S.; Writing-Review \& Editing, G.Z.Funding: This research was funded by National Natural Science Foundation of China [21472041].

Conflicts of Interest: The authors declare no conflict of interest. 


\section{References}

1. Hie, L.; Nathel, N.F.F.; Shah, T.K.; Baker, E.L.; Hong, X.; Yang, Y.-F.; Liu, P.; Houk, K.N.; Garg, N.K. Conversion of amides to esters by the nickel-catalysed activation of amide C-N bonds. Nature 2015, 524, 79-83. [CrossRef] [PubMed]

2. Meng, G.; Szostak, M. Sterically controlled Pd-catalyzed chemoselective ketone synthesis via N-C cleavage in twisted amides. Org. Lett. 2015, 17, 4364-4367. [CrossRef] [PubMed]

3. Li, X.; Zou, G. Acylative Suzuki coupling of amides: Acyl-nitrogen activation via synergy of independently modifiable activating groups. Chem. Commun. 2015, 51, 5089-5092. [CrossRef] [PubMed]

4. Pace, V.; Holzer, W.; Meng, G.; Shi, S.; Lalancette, R.; Szostak, R.; Szostak, M. Structures of highly twisted amides relevant to amide N-C cross-coupling: Evidence for ground-state amide destabilization. Chem. Eur. J. 2016, 22, 14494-14498. [CrossRef] [PubMed]

5. Osumi, Y.; Liu, C.; Szostak, M. N-Acylsuccinimides: Twist-controlled, acyl-transfer reagents in Suzuki-Miyaura cross-coupling by N-C amide bond activation. Org. Biomol. Chem. 2017, 15, 8867-8871. [CrossRef] [PubMed]

6. Cui, M.; Chen, Z.; Liu, T.; Wang, H.; Zeng, Z. N-Acylsuccinimides: Efficient acylative coupling reagents in palladium-catalyzed Suzuki coupling via C-N cleavage. Tetrahedron Lett. 2017, 58, 3819-3822. [CrossRef]

7. Boit, T.B.; Weires, N.A.; Kim, J.; Garg, N.K. Nickel-catalyzed Suzuki-Miyaura coupling of aliphatic amides. ACS Catal. 2018, 8, 1003-1008. [CrossRef] [PubMed]

8. Hie, L.; Baker, E.L.; Anthony, S.M.; Desrosiers, J.-N.; Senanayake, C.; Garg, N.K. Nickel-catalyzed esterification of aliphatic amides. Angew. Chem. Int. Ed. 2016, 55, 15129-15132. [CrossRef] [PubMed]

9. Lanigan, R.M.; Sheppard, T.D. Recent developments in amide synthesis: Direct amidation of carboxylic acids and transamidation reactions. Eur. J. Org. Chem. 2013, 7453-7465. [CrossRef]

10. Baker, E.L.; Yamano, M.M.; Zhou, Y.; Anthony, S.M.; Garg, N.K. A two-step approach to achieve secondary amide transamidation enabled by nickel catalysis. Nat. Commun. 2016, 7, 11554. [CrossRef] [PubMed]

11. Lei, P.; Meng, G.; Ling, Y.; An, J.; Nolan, S.P.; Szostak, M. General method for the Suzuki-Miyaura cross-coupling of primary amide-derived electrophiles enabled by $[\mathrm{Pd}(\mathrm{NHC})(\mathrm{cin}) \mathrm{Cl}]$ at room temperature. Org. Lett. 2017, 19, 6510-6513. [CrossRef] [PubMed]

12. Shi, S.; Szostak, M. Nickel-catalyzed diaryl ketone synthesis by N-C cleavage: Direct Negishi cross-coupling of primary amides by site-selective N, N-di-Boc activation. Org. Lett. 2016, 18, 5872-5875. [CrossRef] [PubMed]

13. Meng, G.; Szostak, M. Site-selective C-H/C-N activation by cooperative catalysis: Primary amides as arylating reagents in directed C-H arylation. ACS Catal. 2017, 7, 7251-7256. [CrossRef]

14. Liu, C.; Liu, Y.; Liu, R.; Lalancette, R.; Szostak, R.; Szostak, M. Palladium-catalyzed Suzuki-Miyaura cross-coupling of N-mesylamides by N-C cleavage: Electronic effect of the mesyl group. Org. Lett. 2017, 19, 1434-1437. [CrossRef] [PubMed]

15. Wang, C.; Huang, L.; Wang, F.; Zou, G. Highly efficient synthesis of aryl ketones by PEPPSI-palladium catalyzed acylative Suzuki coupling of amides with diarylborinic acids. Tetrahedron Lett. 2018, 59, 2299-2301. [CrossRef]

16. Liu, C.; Meng, G.; Liu, Y.; Liu, R.; Lalancette, R.; Szostak, R.; Szostak, M. N-Acylsaccharins: Stable electrophilic amide-based acyl transfer reagents in Pd-catalyzed Suzuki-Miyaura coupling via N-C cleavage. Org. Lett. 2016, 18, 4194-4197. [CrossRef] [PubMed]

17. Wu, H.; Li, Y.; Cui, M.; Jian, J.; Zeng, Z. Suzuki coupling of amides via palladium-catalyzed C-N cleavage of N-acylsaccharins. Adv. Synth. Catal. 2016, 358, 3876-3880. [CrossRef]

18. Liu, C.; Meng, G.; Szostak, M. N-Acylsaccharins as amide-based arylating reagents via chemoselective N-C cleavage: Pd-catalyzed decarbonylative Heck reaction. J. Org. Chem. 2016, 81, 12023-12030. [CrossRef] [PubMed]

19. Cui, M.; Wu, H.; Jian, J.; Wang, H.; Liu, C.; Daniel, S.; Zeng, Z. Palladium-catalyzed Sonogashira coupling of amides: Access to ynones via C-N bond cleavage. Chem. Commun. 2016, 52, 12076-12079. [CrossRef] [PubMed]

20. Wu, H.; Liu, T.; Cui, M.; Li, Y.; Jian, J.; Wang, H.; Zeng, Z. Rhodium-catalyzed C-H functionalization with N-acylsaccharins. Org. Biomol. Chem. 2017, 15, 536-540. [CrossRef] [PubMed] 
21. Meng, G.; Szostak, R.; Szostak, M. Suzuki-Miyaura cross-coupling of N-acylpyrroles and pyrazoles: Planar, electronically activated amides in catalytic N-C cleavage. Org. Lett. 2017, 19, 3596-3599. [CrossRef] [PubMed]

22. Meng, G.; Lalancette, R.; Szostak, R.; Szostak, M. N-methylamino pyrimidyl amides (MAPA): Highly reactive, electronically-activated amides in catalytic N-C(O) cleavage. Org. Lett. 2017, 19, 4656-4659. [CrossRef] [PubMed]

23. Masson-Makdissi, J.; Vandavasi, J.K.; Newman, S.G. Switchable selectivity in the Pd-catalyzed alkylative cross-coupling of esters. Org. Lett. 2018, 20, 4094-4098. [CrossRef] [PubMed]

24. Liu, X.; Jia, J.; Rueping, M. Nickel-catalyzed C-O bond-cleaving alkylation of esters: Direct replacement of the ester moiety by functionalized alkyl chains. ACS Catal. 2017, 7, 4491-4496. [CrossRef]

25. Chatupheeraphat, A.; Liao, H.-H.; Srimontree, W.; Guo, L.; Minenkov, Y.; Poater, A.; Cavallo, L.; Rueping, M. Ligand-controlled chemoselective $\mathrm{C}($ acyl $)-\mathrm{O}$ Bond vs $\mathrm{C}($ aryl $)-\mathrm{C}$ bond activation of aromatic esters in nickel catalyzed $\mathrm{C}\left(\mathrm{sp}^{2}\right)-\mathrm{C}\left(\mathrm{sp}^{3}\right)$ cross-couplings. J. Am. Chem. Soc. 2018, 140, 3724-3735. [CrossRef] [PubMed]

26. Simmons, B.J.; Weires, N.A.; Dander, J.E.; Garg, N.K. Nickel-catalyzed alkylation of amide derivatives. ACS Catal. 2016, 6, 3176-3179. [CrossRef]

27. Liu, X.; Hsiao, C.-C.; Guo, L.; Rueping, M. Cross-coupling of amides with alkylboranes via nickel-catalyzed C-N bond cleavage. Org. Lett. 2018, 20, 2976-2979. [CrossRef] [PubMed]

28. Li, X.; Zou, G. Palladium-catalyzed acylative cross-coupling of amides with diarylborinic acids and sodium tetraarylborates. J. Organomet. Chem. 2015, 794, 136-145. [CrossRef]

29. Si, S.; Wang, C.; Zhang, N.; Zou, G. Palladium-catalyzed room-temperature acylative Suzuki coupling of high-order aryl borons with carboxylic acids. J. Org. Chem. 2016, 81, 4364-4370. [CrossRef] [PubMed]

30. O’Brien, C.J.; Kantchev, E.A.B.; Valente, C.; Hadei, N.; Chass, G.A.; Lough, A.; Hopkinson, A.C.; Organ, M.G. Easily prepared air- and moisture-stable $\mathrm{Pd}-\mathrm{NHC}(\mathrm{NHC}=\mathrm{N}$-heterocyclic carbene) complexes: A reliable, user-friendly, highly active palladium precatalyst for the Suzuki-Miyaura reaction. Chem. Eur. J. 2006, 12, 4743-4748. [CrossRef] [PubMed]

31. Fortman, G.C.; Nolan, S.P. N-Heterocyclic carbene (NHC) ligands and palladium in homogeneous cross-coupling catalysis: A perfect union. Chem. Soc. Rev. 2011, 40, 5151-5169. [CrossRef] [PubMed]

32. Huang, J.; Nolan, S.P. Efficient cross-coupling of aryl chlorides with aryl Grignard reagents (Kumada reaction) mediated by a palladium/imidazolium chloride system. J. Am. Chem. Soc. 1999, 121, 9889-9890. [CrossRef]

33. Kantchev, E.A.B.; Ying, J.Y. Practical one-pot, three-component synthesis of N-heterocyclic carbene (NHC) ligated palladacycles derived from $N, N$-dimethylbenzylamine. Organometallics 2009, 28, 289-299. [CrossRef]

34. Brahmachari, G. Room Temperature Organic Synthesis; Elsevier: Amsterdam, The Netherlands, 2015; ISBN 9780128011386.

35. Brown, H.C.; Sharp, R.L. Hydroboration. XXIV. Directive effects in the hydroboration of some substituted styrenes. J. Am. Chem. Soc. 1966, 88, 5851-5854. [CrossRef]

36. Brown, H.C.; Racherla, U.S. Organoboranes. 44. A convenient, highly efficient synthesis of triorganylboranes via a modified organometallic route. J. Org. Chem. 1986, 51, 427-432. [CrossRef]

37. Samanta, S.; Mishra, B.K.; Pace, T.C.S.; Sathyamurthy, N.; Bohne, C.; Moorthy, J.N. $\beta$-Phenyl quenching of triplet excited ketones: How critical is the geometry for deactivation? J. Org. Chem. 2006, 71, 4453-4459. [CrossRef] [PubMed]

38. Rao, M.L.N.; Venkatesh, V.; Banerjee, D. Atom-efficient cross-coupling reactions of triarylbismuths with acyl chlorides under $\operatorname{Pd}(0)$ catalysis. Tetrahedron 2007, 63, 12917-12926. [CrossRef]

39. Vu, M.D.; Das, M.; Liu, X.-W. Direct aldehyde $\mathrm{C}_{\mathrm{sp}}{ }^{2}-\mathrm{H}$ functionalization through visible light mediated photoredox catalysis. Chem. Eur. J. 2017, 23, 15899-15902. [CrossRef] [PubMed]

40. Zimbron, J.M.; Seeger-Weibel, M.; Hirt, H.; Gallou, F. Development of a robust and practical process for the Darzens condensation and $\alpha, \beta$-epoxide rearrangement: Scope and limitations of the methodology. Synthesis 2008, 8, 1221-1226. [CrossRef]

41. Liu, M.; Hyder, Z.; Sun, Y.; Tang, W.; Xu, L.; Xiao, J. Efficient synthesis of alkyl aryl ketones \& ketals via palladium-catalyzed regioselective arylation of vinyl ethers. Org. Biomol. Chem. 2010, 8, 2012-2015. [CrossRef] [PubMed]

42. Suchand, B.; Satyanarayana, G. KOtBu-mediated domino isomerization and functionalization of aromatic allylic alcohols. Eur. J. Org. Chem. 2017, 3886-3895. [CrossRef] 
43. Uyanik, M.; Suzuki, D.; Yasui, T.; Ishihara, K. In situ generated (hypo)iodite catalysts for the direct

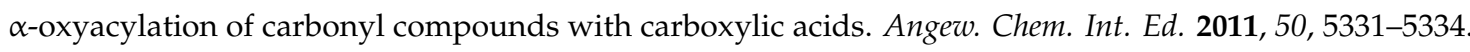
[CrossRef] [PubMed]

44. Li, X.; Li, L.; Tang, Y.; Ling, Z.; Cun, L.; Zhu, J.; Liao, J.; Deng, J. Chemoselective conjugate reduction of $\alpha$, $\beta$-unsaturated ketones catalyzed by rhodium amido complexes in aqueous media. J. Org. Chem. 2010, 75, 2981-2988. [CrossRef] [PubMed]

45. Molander, G.A.; Petrillo, D.E. Suzuki-Miyaura cross-coupling of potassium trifluoroboratohomoenolates. Org. Lett. 2008, 10, 1795-1798. [CrossRef] [PubMed]

46. Colbon, P.; Ruan, J.; Purdie, M.; Xiao, J. Direct acylation of aryl chlorides with aldehydes by palladium-pyrrolidine co-catalysis. Org. Lett. 2010, 12, 3670-3673. [CrossRef] [PubMed]

47. Vautravers, N.R.; Regent, D.D.; Breit, B. Inter- and intramolecular hydroacylation of alkenes employing a bifunctional catalyst system. Chem. Commun. 2011, 47, 6635-6637. [CrossRef] [PubMed]

48. Malanga, C.; Aronica, L.A.; Lardicci, L. Direct $\mathrm{Ni}^{\mathrm{O}}$ mediated synthesis of ketones from acyl bromides and Grignard reagents. Tetrahedron Lett. 1995, 36, 9185-9188. [CrossRef]

Sample Availability: Samples of the compounds are available from the authors. 